\title{
Glassy behavior of a homopolymer from molecular dynamics simulations
}

\author{
Nikolay V. Dokholyan ${ }^{1,2}$, Estelle Pitard ${ }^{3}$, \\ Sergey V. Buldyrev ${ }^{1}$, and H. Eugene Stanley ${ }^{1}$ \\ ${ }^{1}$ Center for Polymer Studies, Physics Department, Boston \\ University, Boston, MA 02215, USA \\ ${ }^{2}$ Department of Chemistry and Chemical Biology, Harvard \\ University, 12 Oxford Street, Cambridge, MA 02138, USA \\ ${ }^{3}$ Laboratoire de Physique Théorique et Mathématique, \\ Université Montpellier II, 34000 Montpellier, France
}

\begin{abstract}
We study at- and out-of-equilibrium dynamics of a single homopolymer chain at low temperature using molecular dynamics simulations. The main quantities of interest are the average root mean square displacement of the monomers below the theta point, and the structure factor, as a function of time. The observation of these quantities show a close resemblance to those measured in structural glasses and suggest that the polymer chain in its low temperature phase is in a glassy phase, with its dynamics dominated by traps. In equilibrium, at low temperature, we observe the trapping of the monomers and a slowing down of the overall motion of the polymer as well as non-exponential relaxation of the structure factor. In out-of-equilibrium, at low temperatures, we compute the two-time quantities and observe breaking of ergodicity in a range of waiting times, with the onset of aging.
\end{abstract}

The problem of the dynamics of a single polymer chain has been addressed in many ways, the simplest theory being the Rouse model [1]. When the polymer is not a Gaussian chain, but evolves either to a swollen or collapsed 
state after a change of external conditions, the dynamics can also be studied using more or less elaborate scenarios, either phenomenologically (like the "sausage" or "necklace" pictures of de Gennes and others [2, 3]), numerically [4] or analytically [5, 6]. Phenomena similar to polymer collapse can be commonly observed as a protein folds into its compact native state structure, or unfolds to an expanded coil (though the protein problem is much more complex than the homopolymer case); more generally a polymer collapses when it is put in a poor solvent, and expands in a good solvent. However, testing the theoretical results with experiments has, thus far, proven difficult, and one has still little insight as to what happens at a microscopic level during such dynamics. It is also expected that the dynamics at low temperatures is slowed down by traps created by the entanglements of the chain, and the compactness of the structure in the collapsed phase. We address this issue here for the first time and show that these effects can be measured through the one-time and two-time dependence of the structure factor; we prove that aging is an intrinsic feature of the dynamics of a long collapsed polymer chain.

We perform the kinetic study of the collapse of a single homopolymer chain after a temperature quench, obtained using molecular dynamics simulations. We characterize the different steps that occur during such collapse. We show how these steps can be interpreted on a microscopic level, especially, how to account for the fact that for an intermediate time regime the dynamics slows down drastically in a way reminiscent of glassy dynamics. We present results for one- and two-time quantities and show that, at low temperatures, the system behaves in an out-of-equilibrium way. Finally, we demonstrate the similarities of a single homopolymer chain with numerical results obtained on polymeric systems, such as polymer melts [7], or glass-formers [16, 17], with the glassy dynamics observed in a one-dimensional model of homopolymer collapse [12], or in 3D heteropolymer and homopolymer dynamics studied via analytical techniques [13].

We study a "beads on a string" model of a homopolymer in $3 \mathrm{D}$, consisting of $N=256$ monomers. We model the monomers as hard spheres of unit mass with a square-well attractive potential between every pair of contacts. The total potential energy of the polymer is

$$
\mathcal{E}=\frac{1}{2} \sum_{i \neq j=1}^{N} U_{i j}+\sum_{i=1}^{N-1} V_{i i+1},
$$


where

$$
U_{i j}= \begin{cases}+\infty, & \left|\mathbf{r}_{i}-\mathbf{r}_{j}\right| \leq a_{0} \\ -\epsilon, & a_{0}<\left|\mathbf{r}_{i}-\mathbf{r}_{j}\right| \leq a_{1} \\ 0, & \left|\mathbf{r}_{i}-\mathbf{r}_{j}\right|>a_{1}\end{cases}
$$

is the matrix of interactions between monomers $i$ and $j$, and

$$
V_{i+1}= \begin{cases}0, & a_{0}<\left|\mathbf{r}_{i}-\mathbf{r}_{i+1}\right|<a_{1} \\ +\infty, & \left|\mathbf{r}_{i}-\mathbf{r}_{i+1}\right| \leq a_{0}, \text { or }\left|\mathbf{r}_{i}-\mathbf{r}_{i+1}\right| \geq a_{1}\end{cases}
$$

is the potential energy of covalent bonds. $a_{0} / 2$ is the radius of the hard sphere, and $a_{1} / 2$ is the radius of the attractive sphere $\left(\epsilon=1, a_{0}=8.00\right.$ and $\left.a_{1}=11.28\right)$. The $\theta$-point temperature for the homopolymer studied is $T_{\theta} \approx 1.75$. The average radius of gyration of the collapsed globule at $T=0.2 \ll T_{\theta}$ is $R_{G} \approx 24.7$.

We employ the discrete molecular dynamics algorithm [14. To control the temperature of the homopolymer, we introduce a heat bath of 2746 particles, which do not interact with the homopolymer or with each other in any way but via collisions 15. By changing the kinetic energy of heat bath particles we control the temperature of the environment. The heat bath particles are hard spheres of the same radii as the monomers and have unit mass. The temperature units are in $\epsilon / k_{B}$. The time unit (see [15] for details) is estimated from the shortest time between two consequent collisions in the system between any two particles.

Next, we compute: (A) the root mean square displacement (rmsd) $R\left(t_{w}, t_{w}+\right.$ $\tau)$ of the polymer between the two times $t_{w}$ and $t_{w}+\tau$ :

$$
R\left(t_{w}, t_{w}+\tau\right)=\left\langle\left[\frac{1}{N} \sum_{i=1}^{N}\left(\mathbf{r}_{i}\left(t_{w}\right)-\mathbf{r}_{i}\left(t_{w}+\tau\right)\right)^{2}\right]^{1 / 2}\right\rangle,
$$

where $\mathbf{r}_{i}\left(t_{w}\right)$ and $\mathbf{r}_{i}\left(t_{w}+\tau\right)$ are the coordinates of the monomers at times $t_{w}$ and $t_{w}+\tau$. The coordinates at times $t_{w}$ and $t_{w}+\tau$ are adjusted with respect to each other so that: (i) homopolymer's centers of mass overlap at these two times; (ii) their relative orientation is modified using Kabsch transformation [15]. (B) The dynamic structure factor is defined as

$$
F_{q}\left(t_{w}, t_{w}+\tau\right) \equiv 1 / N\left\langle\rho_{q}^{*}\left(t_{w}\right) \rho_{q}\left(t_{w}+\tau\right)\right\rangle,
$$

where $\rho_{q}(t) \equiv \sum_{i} \exp \left(i \mathbf{q} \cdot \mathbf{r}_{i}(t)\right)$. We compute this quantity for $q=q_{\max }$ which corresponds to the maximum of the equilibrium structure factor and 
is equal in our system to $q_{\max }=12 / 90$. Thermal averaging, $\langle\ldots\rangle$, is done over 10 initial configurations.

First, we study the polymer at equilibrium, which is achieved by simulating the polymer at fixed target temperature for approximately $10^{6}$ time units. In this case, we expect time-translational invariance for $R\left(t_{w}, t_{w}+\tau\right)=R(\tau)$ and $F_{q}\left(t_{w}, t_{w}+\tau\right)=F_{q}(\tau)$. We simulate the polymer at various temperatures ranging from 0.2 to 1.75. We find that there are three distinct regions for the $R(\tau)$ dependence on $\tau$ at low temperatures (Fig. 1). The first region can be viewed as a ballistic motion of the monomers until they reach their nearest neighbors. Indeed, we find that the slope of $R(\tau)$ vs $\tau$ on $\log$-log scale is close to 1 in this region. Starting at around $\tau \sim 10$, the rmsd dependence on $\tau$ becomes slower. The lower the temperature, the flatter is the plot of $R(\tau)$ vs $\tau$ in this region. Following the dynamics of a single monomer we can explain the slowing down of $R(\tau)$ by the presence of neighbors which trap this monomer in a "cage". To escape the trap, a collective effort of the monomer and its neighbors is necessary. Thus, this region can be seen as a regime of activated processes that drive monomers beyond their cages. At later times the dynamics of the system becomes diffusive, where we expect and find $R(\tau) \sim \sqrt{\tau}$, except for $T=0.2$ where equilibrium could not be achieved during the simulation time. With the increase of temperature the trapping time becomes smaller and we observe a change in the curvature of the plot $R(\tau)$ vs $\tau$, where the trapping plateau actually disappears. The change of curvature occurs at a temperature around $T^{*} \approx 0.7$.

In order to compare the dynamic behavior of our homopolymer with that of known glassy systems (see e.g. [16, 17]), we study the structure factor $F_{q}(\tau)$ at equilibrium for various temperatures (Fig. 2). The mode coupling theory (MCT) for glasses [9], has shown that in such systems, the scaling of the structure factor behaves non-exponentially with time and predicts powerlaw or stretched-exponential dependence according to the time regime. We find that $F_{q}(\tau)$ has the properties predicted by MCT at low temperatures (see Fig. 2): (i) initially $F_{q}(\tau)$ decays as a power-law function $1-F_{q}(\tau) / F_{q}(0) \sim$ $\tau^{-g}$ to a "plateau", $F_{q}^{c}$; (ii) $F_{q}(\tau)$ approaches the plateau, $F_{q}^{c}$, as a powerlaw function $\left(F_{q}(\tau)-F_{q}^{c} \sim\left(\tau / \tau_{0}\right)^{-a}\right)$; (iii) $F_{q}(\tau)$ departs from the plateau as a power-law, $F_{q}^{c}-F_{q}(\tau) \sim\left(\tau / \tau^{*}\right)^{b}$ ( $\beta$-relaxation); (iv) in a larger time sale $(\alpha$-relaxation $) F_{q}(\tau)$ decays to 0 as a stretched exponential, $F_{q}(\tau) \sim$ $\exp \left(-\left(\tau / \tau^{*}\right)^{\beta}\right)$. 
We find that $(i)$ the exponent $g=1.9 \pm 0.2$, which is in agreement with the expected exponent $g=2$ due to the ballistic motion of the particles at the time scales equal to the size of a typical cage divided by the typical velocity of a particle; (ii) exponent $a, 0.3<a<0.7$, is difficult to determine due to uncertainty in $F_{q}^{c} / F_{q}(0) \approx 0.8-0.9$; (iii) the departure from the plateau, $F_{q}^{c} \approx 0.9$, is well fitted by a power-law with the exponent $b=0.3 \pm 0.02$, which is almost independent of $T$; (iv) the $\alpha$-relaxation is well fitted by a stretched exponential with the exponent $\beta=0.52 \pm 0.03$, which is also independent of $T$ [18.

The sign that a system is of out-of-equilibrium is the violation of timetranslational invariance of quantities such as $R\left(t_{w}, t_{w}+\tau\right)$ and $F_{q}\left(t_{w}, t_{w}+\tau\right)$, called aging [10]. To recover aging phenomena, we study the dependence of $R\left(t_{w}, t_{w}+\tau\right)$ and $F_{q}\left(t_{w}, t_{w}+\tau\right)$ on $t_{w}$. We quench the polymer from a high temperature $\left(T=T_{\theta}\right)$ to lower target temperatures $T_{t}$ and then let the polymer evolve at $T_{t}$. We start computing the two-time quantities after some waiting time $t_{w}$ after the quench (Figs. 3 and 4).

We find that $R\left(t_{w}, t_{w}+\tau\right)$ and $F_{q}\left(t_{w}, t_{w}+\tau\right)$ do not exhibit dependence on $t_{w}$ (see Figs. 3a and $4 \mathrm{a}$ ) if we quench the polymer to temperatures above $T_{t}=T_{b} \approx 0.6$. At $T_{b}=T_{t} \approx 0.6, R\left(t_{w}, t_{w}+\tau\right)$ and $F_{q}\left(t_{w}, t_{w}+\tau\right)$ become dependent on $t_{w}$, indicating a slowing down of the dynamics as $t_{w}$ increases. Thus, $T_{b}$ is the temperature at which we observe the breaking of the time translational invariance. At $T_{t}=0.3$ (see Figs. $3 \mathrm{~b}$ and $4 \mathrm{~b}$ ) we observe a separation of the $R\left(t_{w}, t_{w}+\tau\right)$ and $F_{q}\left(t_{w}, t_{w}+\tau\right)$ vs $\tau$ curves with respect to waiting time $t_{w}$. In the large $t_{w}$ and $\tau$ regime, we show that both $R\left(t_{w}, t_{w}+\tau\right)$ and $F_{q}\left(t_{w}, t_{w}+\tau\right)$ can be rescaled as a function of $\tau / t_{w}^{\mu}$ (see Figs $3 \mathrm{~b}$ and $4 \mathrm{~b}$ ). The exponent $\mu$ is equal to 0.45 for $T_{t}=0.3$ and is the same for $R\left(t_{w}, t_{w}+\tau\right)$ and $F_{q}\left(t_{w}, t_{w}+\tau\right) . \mu$ depends strongly on the temperature and it increases as the temperature decreases.

In a compact state (at $T_{t} \approx 0.3$ ), the polymer exhibits glassy behavior on the time scale accessible to our simulations. The spatial rearrangements of monomers are slower due to the existence of energetic barriers. Due to the finite length of our homopolymer, we expect that at long times, equilibrium is achieved and the time-translational invariance is restored.

We find that the dynamics of a polymer chain at low temperatures is similar to the dynamics of strongly frustrated systems, such as glasses [8], and of strongly disordered systems, such as spin-glasses [10. Equilibrium plots similar to Fig. 1 and Fig. 2 have been previously found in studies of 
polymer melts [7]. Here we also demonstrate that the existence of aging is an intrinsic feature of the dynamics of a polymer chain at low temperatures. We expect the microscopic mechanisms responsible for the slowing down of the dynamics to be similar to those found in glasses, and they can be easily identified in our case as geometrical frustration. The two temperatures, $T^{*} \approx 0.7$ and $T_{b} \approx 0.6$, at which trapping starts to dominate the dynamics and time translational invariance is broken, are close to each other and may well correspond to a unique dynamical transition temperature. The analysis of the equilibrium data is consistent with some of the results predicted by the MCT theory. From the point of view of aging, we do not know any theory for 3D polymer dynamics that would predict a $\tau / t_{w}^{\mu}$ scaling. However, this scaling (with $\mu<1$ ) can be found in a number of systems, including real spin-glasses [10], and in models of traps [11] or in a model of a onedimensional polymer [12]; whereas a $\tau / t_{w}$ scaling is more specific to nearmean-field models [10, 13].

A single homopolymer provides a new example of a non-disordered system where geometry alone induces slow relaxation and aging. A number of polymeric systems in a compact state (single polymeric molecules, polymer melts, collapsed proteins) share the dynamical features described here. Some open questions remain, such as the very long time regime of the relaxation which is hard to characterize due to long computational times.

We thank A. Coniglio, W. Kob, S. Mossa, F. Sciortino, and E. I. Shakhnovich for helpful discussions. NVD is supported by NIH postdoctoral fellowship GM20251-01. EP is supported by NIH grant GM 52126 and by Centre National de la Recherche Scientifique (France).

\section{References}

[1] P. G. de Gennes, Scaling Concepts in Polymer Physics, Cornell University Press (1979).

[2] P. G. de Gennes, J. Phys. Lett. 46, L639 (1985); A. Buguin, F. BrochardWyart and P. G. de Gennes, C. R. Acad. Sci. Paris, 322, Série II b, 741 (1996).

[3] A. Halperin and P. M. Goldbart, Phys. Rev. E 61, 565 (2000). 
[4] G. E. Crooks, B. Ostrowsky and Y. Bar-Yam, Phys. Rev. E 60, 4559 (1999).

[5] E. Pitard and H. Orland, Europhys. Lett. 41, 467 (1998); E. Pitard, Eur. Phys. J. B 7, 665 (1999).

[6] Y. A. Kuznetsov, E. G. Timoshenko and K. A. Dawson, J. Chem. Phys. 104, 3338 (1996) and references therein.

[7] J. Baschnagel, C. Bennemann, W. Paul and K. Binder, J. Phys: Cond. Matt., 11, A47 (1999).

[8] W. Kob, in Soft and Fragile Matter, M. E. Cates and M. Evans (ed.), IOP (2000).

[9] W. Gotze, in Les Houches 1990: Liquids, Freezing and the Glass Transition, part 1, ed. J.-P. Hansen, D. Levesque and J. Zinn-Justin (North Holland, Amsterdam).

[10] J.-P. Bouchaud, L. F. Cugliandolo, J. Kurchan and M. Mézard, in Spin Glasses and Random Fields, A. P. Young (ed.), World Scientific, Singapore (1997).

[11] J.-P. Bouchaud, in Soft and Fragile Matter, M. E. Cates ans M. Evans (ed.), IOP (2000).

[12] E. Pitard and J.-P. Bouchaud, Eur. Phys. J. E 5, 133-148 (2001).

[13] E. Pitard and E. I. Shakhnovich, Phys. Rev. E 63, 041501 (2001).

[14] A. Yu. Grosberg, and A. R. Khokhlov, in Giant Molecules, Academic Press, Boston (1997).

[15] N. V. Dokholyan, S. V. Buldyrev, H. E. Stanley, and E. I. Shakhnovich, Folding \& Design 3, 577 (1998).

[16] F. Sciortino, P. Gallo, P. Tartaglia and S.-H. Chen, Phys. Rev. E 54, 6331 (1996).

[17] S. Mossa, R. Di Leonardo, G. Ruocco, \& M. Sampoli, Phys. Rev. E 62, $612(2000)$. 
[18] Another prediction of the MCT is that the relaxation time $\tau^{*}$ during the $\alpha$-relaxation scales with the temperature as $\tau^{*} \simeq \tau_{0}\left|T-T_{c}\right|^{-\gamma}$. The exponent $\gamma$ is not independent but is related to exponents $a$ and $b$ as $\gamma=1 / 2 a+1 / 2 b$. We estimate the exponent $\gamma=5 \pm 3$ and $T_{c}=0.03 \pm 0.01$ from the $F_{q}(\tau)$ dependence on $\tau$ for various $T$.

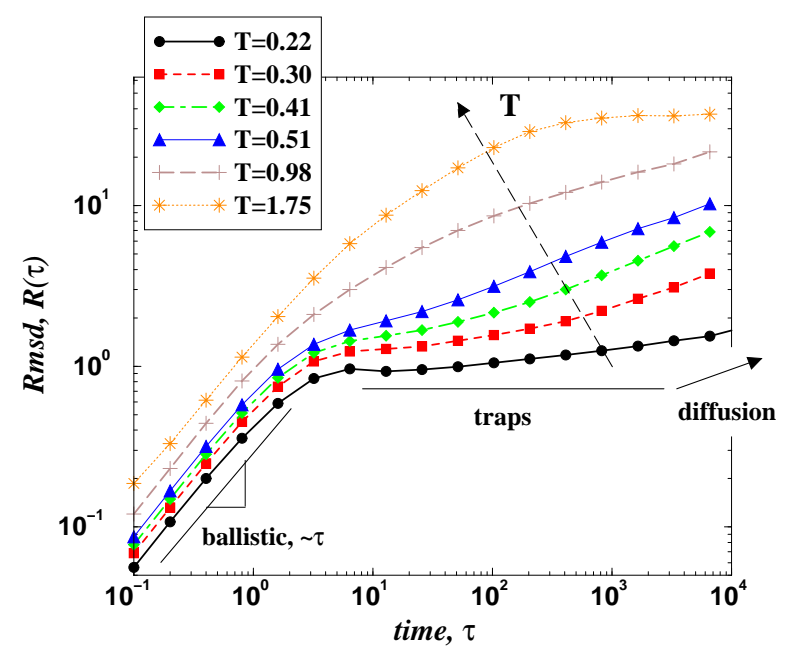

Figure 1: The dependence of the $\operatorname{rmsd} R(\tau)$ on $\tau$ in equilibrium simulations at various fixed temperatures for a polymer chain of 256 monomers. Three regimes can be observed: (i) ballistic (at small time scales), (ii) dominated by traps (at intermediate time scales), and (iii) diffusive (at long time scales). As the temperature increases, the second regime becomes less apparent and, at temperatures around $T^{*} \approx 0.7$, it disappears. 


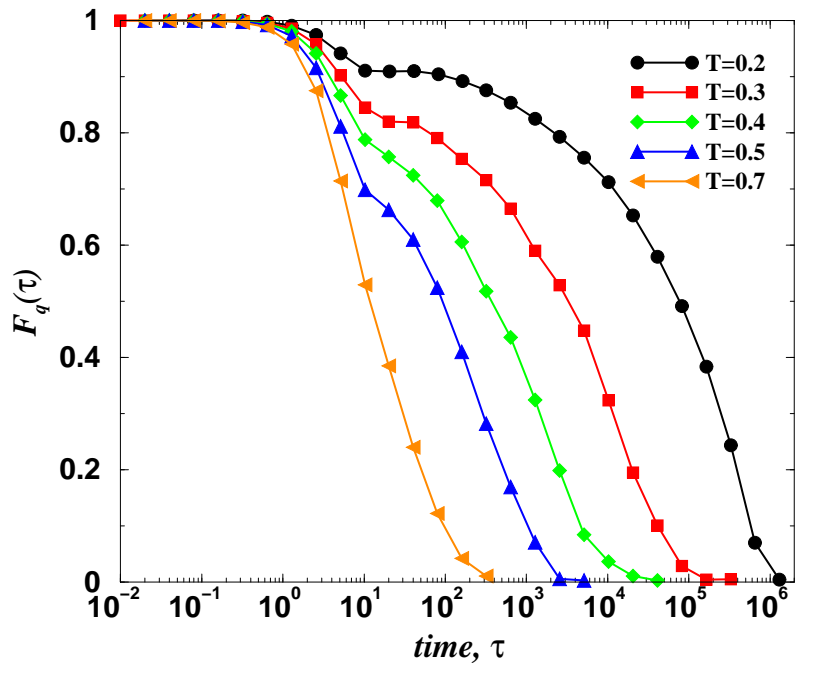

Figure 2: The dependence of the structure function, $F_{q}(\tau)$ on $\tau$ in equilibrium simulations at various fixed temperatures. We choose $q=q_{\max }$, which corresponds to the nearest neighbor shell of the polymer. 

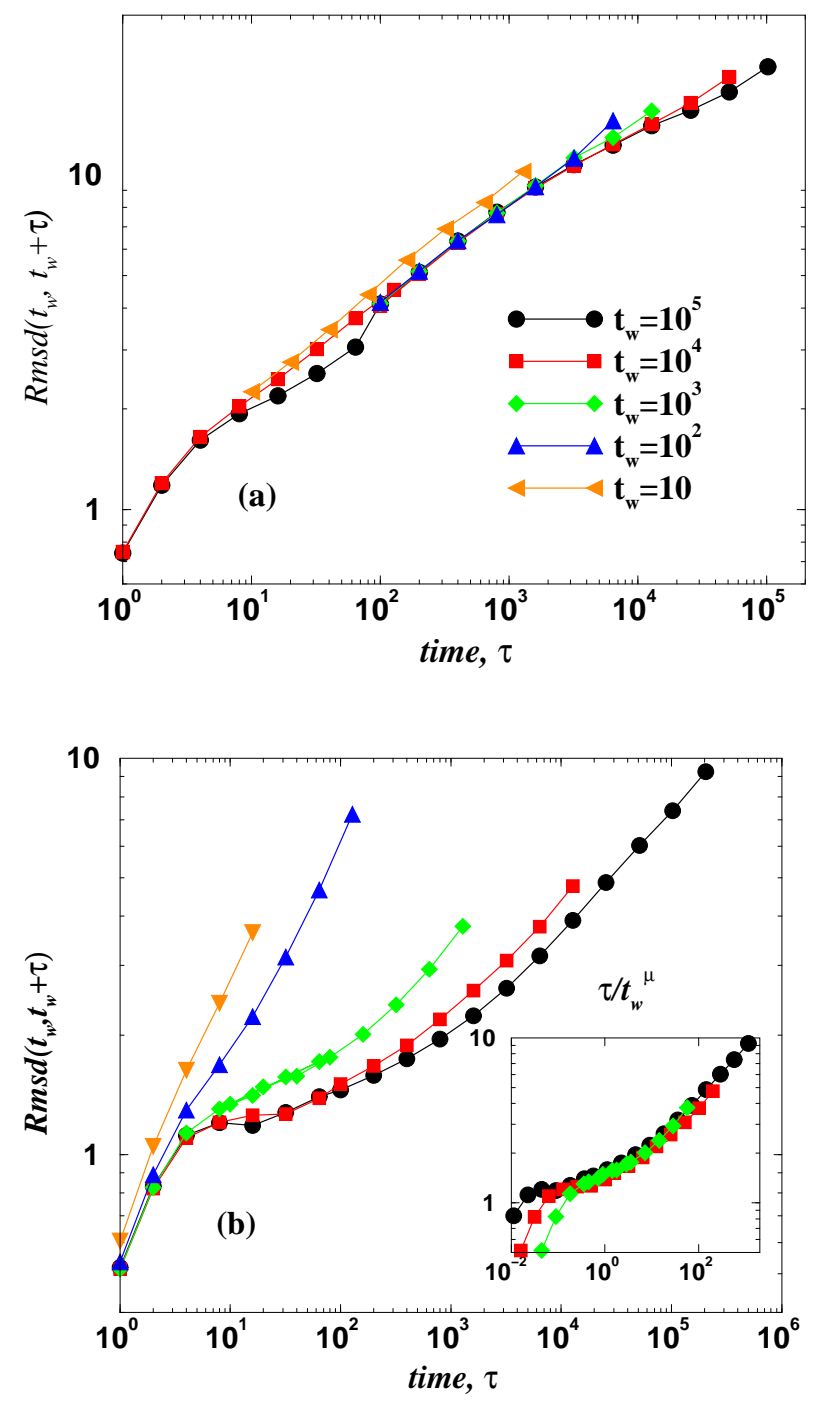

Figure 3: The dependence of the $\operatorname{rmsd} R\left(t_{w}, t_{w}+\tau\right)$ on $\tau$ in quenching simulations from $T_{\theta} \approx 1.75$ to (a) $T_{t}=0.6$ (no aging observed) and (b) $T_{t}=0.3$ (aging) for various waiting times, $t_{w}$. Inset: rescaling of the curves for $t_{w}=10^{5}, 10^{4}, 10^{3}$, with respect to $\tau / t_{w}^{\mu}$, where $\mu \simeq 0.45$. The drastic difference between (a) and (b) points towards the existence of a temperature at which the polymer dynamics becomes non-ergodic. 

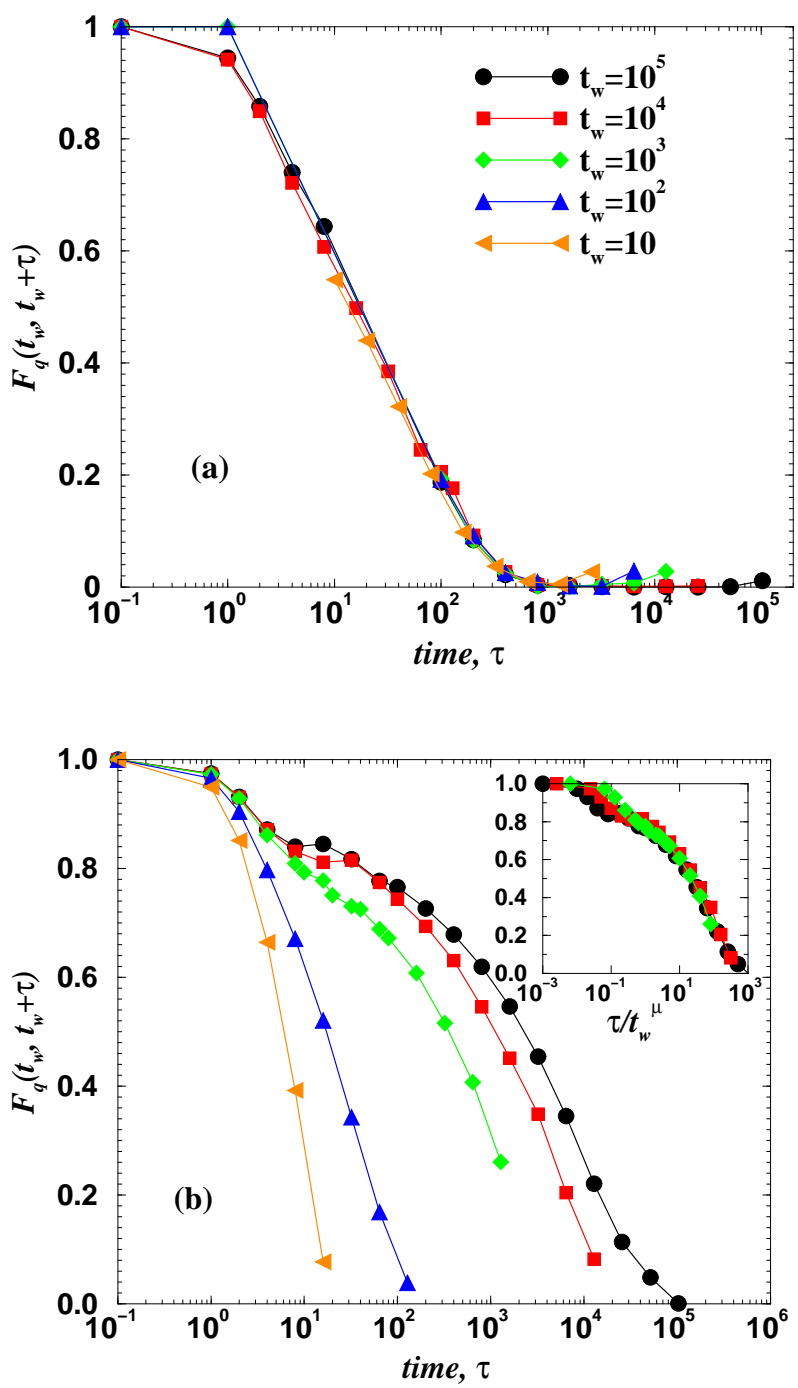

Figure 4: The same as Fig. 3 but for the structure factor $F_{q}\left(t_{w}, t_{w}+\tau\right)$. The exponent $\mu$ is equal to 0.45 . 